Low Temperature Physics/Fizika Nizkikh Temperatur, 2018, v. 44, No.5, pp. 568-572.

\title{
X-ray absorption near edge spectroscopy of thermochromic phase transition in $\mathrm{CuMoO}_{4}$
}

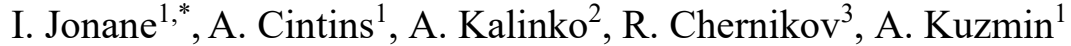 \\ ${ }^{1}$ Institute of Solid State Physics, University of Latvia, Kengaraga street 8, LV-1063 Riga, \\ Latvia \\ ${ }^{2}$ Universität Paderborn, Naturwissenschaftliche Fakultät, Department Chemie, Warburger \\ Strasse 100, 33098 Paderborn, Germany \\ ${ }^{3}$ DESY Photon Science, Notkestraße 85, D-22607 Hamburg, Germany \\ *Corresponding author. E-mail: inga.jonane@cfi.lu.lv
}

Keywords: X-ray absorption spectroscopy, $\mathrm{CuMoO}_{4}$, thermochromism, phase transition PACS: 61.05.cj X-ray absorption spectroscopy: EXAFS, NEXAFS, XANES, etc. 64.70.kp Ionic crystals

\begin{abstract}
Thermochromic phase transition was studied in $\mathrm{CuMoO}_{4}$ using the $\mathrm{Cu}$ and Mo K-edge X-ray absorption spectroscopy in the temperature range of 10-300 K. The hysteretic behavior has been evidenced from the temperature dependence of the pre-edge shoulder intensity at the Mo K-edge, indicating that the transition from brownish-red $\gamma-\mathrm{CuMoO}_{4}$ to green $\alpha-\mathrm{CuMoO}_{4}$ occurs in the temperature range of $230-280 \mathrm{~K}$ upon heating, whereas the $\alpha$-to- $\gamma$ transition occurs between 200 and $120 \mathrm{~K}$ upon cooling. Such behavior of the pre-edge shoulder at the Mo K-edge correlates with the change of molybdenum coordination between distorted tetrahedral in $\alpha-\mathrm{CuMoO}_{4}$ and distorted octahedral in $\gamma-\mathrm{CuMoO}_{4}$. This result has been supported by ab initio full-multiple-scattering X-ray absorption near edge structure (XANES) calculations.
\end{abstract}


Low Temperature Physics/Fizika Nizkikh Temperatur, 2018, v. 44, No.5, pp. 568-572.

\section{Introduction}

Molybdates and tungstates find a wide range of practical applications due to their ability to adopt different crystallographic structures, whose physical properties can be further modified by a chemical composition $[1,2]$. In particular, these materials are used as scintillators, down-conversion phosphors, white light-emitting diodes, laser host materials, catalysts, sensors and pigments [3-12].

Copper molybdate $\left(\mathrm{CuMoO}_{4}\right)$ is a functional oxide material exhibiting thermochromic and piezochromic properties [13-15]. It attracts much attention because of its potential applications for temperature sensing in the ranges where majority of organic compounds and liquid crystals are unstable. Possible chromic-related applications extend from the user-friendly temperature and pressure indicators [14-17] to "smart" inorganic pigments [18]. Besides, copper molybdate has also promising catalytic properties [19-23].

Structural, optical, electrical and magnetic properties of $\mathrm{CuMoO}_{4}$ were previously studied in [13, 24-27]. Currently six different structural phases of $\mathrm{CuMoO}_{4}$ are known [24, 28]. In this study, we focus on two of them - green $\alpha-\mathrm{CuMoO}_{4}$ and brownish-red $\gamma-\mathrm{CuMoO}_{4}$ phases that are stable at high and low temperatures, respectively (Fig. 1).

FIG. 1. Crystallographic structures of high-temperature $\alpha-\mathrm{CuMoO}_{4}$ and low-temperature $\gamma$ $\mathrm{CuMoO}_{4}$ phases [24].

At ambient pressure and room temperature, $\mathrm{CuMoO}_{4}$ has $\alpha$-phase with triclinic structure (space group $\mathrm{P}-1$ ) $[24,29]$. It is composed of distorted $\mathrm{CuO}_{6}$ octahedra, $\mathrm{CuO}_{5}$ square-pyramids and $\mathrm{MoO}_{4}$ tetrahedra. By decreasing temperature below $\sim 200 \mathrm{~K}$ (or by applying pressure above $0.2 \mathrm{GPa}$ at room temperature) reversible first order phase transition from $\alpha-\mathrm{CuMoO}_{4}$ to $\gamma-\mathrm{CuMoO}_{4}$ occurs [24]. Low temperature $\gamma$ modification also has triclinic lattice (space group $P$-1) built up of distorted $\mathrm{CuO}_{6}$ and $\mathrm{MoO}_{6}$ octahedra [25]. The $\alpha$-to- $\gamma$ phase transition is accompanied by color change (from green to brownish-red) and volume reduction of $12-13 \%$ [24] that makes the two phases easily distinguishable. However, the presence in both phases of three non-equivalent $\mathrm{Cu}$ and Mo atoms with different local environment makes structural analysis challenging [24].

The $\alpha$-to- $\gamma$ phase transition occurring below room temperature was studied previously using X-ray diffraction [24] and optical spectroscopy [14, 26], differential scanning 
Low Temperature Physics/Fizika Nizkikh Temperatur, 2018, v. 44, No.5, pp. 568-572.

calorimetry and magnetic susceptibility [14] measurements. It was found that the transition has hysteretic behavior, so that lower temperature is required to promote the $\alpha$-to- $\gamma$ transition than $\gamma$-to- $\alpha$.

Chromic properties of $\mathrm{CuMoO}_{4}$ are associated with the interplay between two optical absorption bands: the one in the blue range (around $3 \mathrm{eV}$ ) is due to the interband transition across the band gap of the crystal and the second one in the red (around $1.49 \mathrm{eV}$ ) originates due to the intraband d-d transition at copper $[13,14,26]$. Note that the thermochromic phase transition is strongly affected by chemical composition: for example, an addition of tungsten to $\mathrm{CuMoO}_{4}$ results in a formation of solid solutions $\mathrm{CuMo}_{1-x} \mathrm{~W}_{x} \mathrm{O}_{4}(x \leq 0.12)$ and shifts the transition temperature by up to $100 \mathrm{~K}[14,18,24]$.

Since thermochromic and piezochromic properties of $\mathrm{CuMoO}_{4}$ are connected with its local structure, X-ray absorption spectroscopy (XAS) is an obvious choice to probe structural changes during the phase transition. XAS provides information complementary to other techniques and, in the case of $\mathrm{CuMoO}_{4}$, can probe the local environment around $\mathrm{Cu}$ and $\mathrm{Mo}$ atoms independently by detecting two different absorption edges. Therefore, we have performed the temperature-dependent $(10-300 \mathrm{~K}) \mathrm{Cu}$ and Mo K-edge XAS study of thermochromic phase transition in $\mathrm{CuMoO}_{4}$ at the new P65 beamline (HASYLAB at DESY, Hamburg) using the synchrotron radiation from the PETRA-III storage ring. The obtained results allowed us to confirm the hysteretic nature of phase transition and to explain the influence of structural changes on the X-ray absorption near edge structure based on ab initio full-multiple-scattering calculations.

\section{Experimental and data analysis}

Polycrystalline $\mathrm{CuMoO}_{4}$ powder was synthesized using solid-state reaction method by heating a mixture of $\mathrm{CuO}$ and $\mathrm{MoO}_{3}$ powders at $650^{\circ} \mathrm{C}$ in air for $8 \mathrm{~h}$ followed by cooling down to room temperature. The as-prepared powder corresponded to $\alpha-\mathrm{CuMoO}_{4}$ phase and had green color.

Temperature-dependent (from 10 to $300 \mathrm{~K}$ ) X-ray absorption experiments were conducted at the HASYLAB PETRA-III P65 undulator beamline. The PETRA-III storage ring operated at $E=6.08 \mathrm{GeV}$ and current $I=95 \mathrm{~mA}$ in top- up 40 bunch mode. The harmonic 
Low Temperature Physics/Fizika Nizkikh Temperatur, 2018, v. 44, No.5, pp. 568-572.

rejection was achieved by uncoated ( $\mathrm{Cu}$ edge) and $\mathrm{Rh}$-coated (Mo edge) silicon plane mirrors. Fixed exit Si(311) double-crystal monochromator was used in all experiments. The X-ray absorption spectra were collected at the $\mathrm{Cu}(8979 \mathrm{eV})$ and Mo $(20000 \mathrm{eV}) \mathrm{K}$-edges in transmission mode using two ionization chambers. The Oxford Instruments liquid helium flow cryostat was used to maintain the sample temperature. The $\mathrm{CuMoO}_{4}$ powder was gently milled in agate mortar and deposited on Millipore membrane.

The $\mathrm{Cu}$ and Mo K-edge XANES parts of the X-ray absorption spectra were isolated and used in the further analysis. It was observed that the $\mathrm{Cu}$ K-edge XANES spectra do not vary significantly upon temperature change indicating some stability of the copper environment. Therefore, they will not be discussed further. At the same time, the Mo K-edge XANES was significantly affected by temperature variation. The experimental $\mathrm{Mo}$ and $\mathrm{Cu}$ K-edge XANES spectra corresponding to $\gamma-\mathrm{CuMoO}_{4}$ at $10 \mathrm{~K}$ and $\alpha-\mathrm{CuMoO}_{4}$ at $300 \mathrm{~K}$ are shown in Figs. 2(a,b). The pre-edge shoulder at the Mo K-edge is clearly visible around $20000 \mathrm{eV}$, and its change can be related to a transition from $\mathrm{MoO}_{4}$ tetrahedra to $\mathrm{MoO}_{6}$ octahedra upon cooling. At the same time, the $\mathrm{Cu}$ K-edge XANES does not change significantly upon phase transition.

FIG. 2. (a,b) Experimental Mo and $\mathrm{Cu} \mathrm{K}$-edge XANES of $\alpha-\mathrm{CuMoO}_{4}$ (at $300 \mathrm{~K}$ ) and $\gamma$ $\mathrm{CuMoO}_{4}$ (at $10 \mathrm{~K}$ ) phases. (c) Calculated Mo K-edge XANES for regular $\mathrm{MoO}_{4}$ tetrahedron, distorted and regular $\mathrm{MoO}_{6}$ octahedra. Numbers in brackets indicate the direction of molybdenum ion off-center displacement. See text for more details.

A fraction of $\alpha-\mathrm{CuMoO}_{4}$ phase was evaluated at each temperature during heating from $10 \mathrm{~K}$ to $300 \mathrm{~K}$ and cooling from $300 \mathrm{~K}$ down to $100 \mathrm{~K}$ using a linear combination of the lowest temperature $(10 \mathrm{~K})$ and highest temperature $(300 \mathrm{~K})$ Mo K-edge XANES. The analysis was performed using Athena package [30] in the energy range from $19980 \mathrm{eV}$ to $20010 \mathrm{eV}$ with the aim to sample a variation of the pre-edge shoulder. The obtained results are shown in Fig. 3, where the hysteretic behavior is well observed.

FIG. 3. Temperature dependence of the fraction of $\alpha-\mathrm{CuMoO}_{4}$ phase upon heating (solid circles) and cooling (open circles). 
Low Temperature Physics/Fizika Nizkikh Temperatur, 2018, v. 44, No.5, pp. 568-572.

\section{XANES calculations}

Full-multiple-scattering (FMS) XANES calculations were performed using ab initio real-space FDMNES code $[31,32]$ employing muffin-tin (10\% overlap) self-consistent potential. The dipole and quadrupole transitions were taken into account, and the energydependent real Hedin-Lundqvist exchange-correlation potential was used [33, 34]. The calculated XANES spectra were broadened to account for the core-hole level width $(5.8 \mathrm{eV}$ at the Mo K-edge [35]) and other multielectronic phenomena. The energy origin was set at the Fermi level $E \mathrm{~F}$.

The results of FMS XANES calculations for five $\mathrm{MoO}_{x}$ clusters are shown in Fig. 2(c). They correspond to regular $\mathrm{MoO}_{4}$ tetrahedron $(R(\mathrm{Mo}-\mathrm{O})=1.78 \AA)$, regular $\mathrm{MoO}_{6}$ octahedron $(R(\mathrm{Mo}-\mathrm{O})=1.98 \AA)$ and distorted $\mathrm{MoO}_{6}$ octahedra with molybdenum ion being displaced in the direction of octahedron apex by $0.2 \AA(<100>)$, to the octahedron edge by $0.28 \AA(<110>)$ and to the octahedron face by $0.35 \AA(<111>)$.

FMS XANES calculations were also performed for $\alpha$ - and $\gamma$-CuMoO 4 structural models created using diffraction data from [24]. The total calculated Mo K-edge XANES spectra and separate contributions from non-equivalent molybdenum atoms (Mo1, Mo2 and Mo3) are compared with the experimental data in Fig. 4.

FIG. 4. Comparison of the experimental and calculated Mo K-edge XANES of $\alpha$-CuMoO 4 and $\gamma-\mathrm{CuMoO}_{4}$ phases (upper panel). Contributions from three non-equivalent molybdenum atoms (Mo1, Mo2, Mo3) to the Mo K-edge XANES are also shown in the middle and lower panels for $\alpha-\mathrm{CuMoO}_{4}$ and $\gamma-\mathrm{CuMoO}_{4}$ phases, respectively.

\section{Results and discussion}

The Mo K-edge XANES spectra of $\mathrm{CuMoO}_{4}$ at two temperatures $(10$ and $300 \mathrm{~K})$ are shown in Fig. 2(a). The pre-edge shoulder at $\sim 20000 \mathrm{eV}$ corresponds to the $1 \mathrm{~s}(\mathrm{Mo}) \rightarrow 4 \mathrm{~d}(\mathrm{Mo})+2 \mathrm{p}(\mathrm{O})$ transition. Note that the final state of the electron is the relaxed excited state in the presence of the core hole at the $1 \mathrm{~s}(\mathrm{Mo})$ level screened by other electrons. The transition is forbidden in the dipole approximation for a regular $\mathrm{MoO}_{6}$ octahedron, having an inversion center, but becomes allowed when inversion symmetry is broken as in 
Low Temperature Physics/Fizika Nizkikh Temperatur, 2018, v. 44, No.5, pp. 568-572.

distorted octahedron [36, 37] or in tetrahedron [38] (Fig. 2(c)). The amplitude of the preedge shoulder depends on the degree of the $\mathrm{MoO}_{6}$ octahedra distortion and $4 \mathrm{~d}(\mathrm{Mo}) / 2 \mathrm{p}(\mathrm{O})$ orbital mixing and is the largest for tetrahedral $\mathrm{MoO}_{4}$ coordination [39]. Therefore, it can be used to monitor the $\gamma$-to- $\alpha$ phase transition in $\mathrm{CuMoO}_{4}$.

Since accurate separation of the pre-edge shoulder from the main absorption edge is tricky, we employed a different approach: the experimental XANES spectrum at each temperature was approximated by a linear combination of the lowest temperature $(10 \mathrm{~K})$ and highest temperature (300 K) XANES spectra. As a result, the fraction of the $\alpha-\mathrm{CuMoO}_{4}$ phase in the total XANES upon heating and cooling was estimated and is reported in Fig. 3. As one can see, only $\gamma-\mathrm{CuMoO}_{4}$ is present below $150 \mathrm{~K}$ and only $\alpha-\mathrm{CuMoO}_{4}$ is observed above $280 \mathrm{~K}$, as expected.

Linear combination analysis showed that upon heating, the $\gamma$-to- $\alpha$ phase transition occurs between $\sim 230-280 \mathrm{~K}$, whereas upon cooling, the $\alpha$-to- $\gamma$ transition takes place at lower temperature between $\sim 120-200 \mathrm{~K}$, showing a large hysteresis loop with the range of two phase coexistence of about 50-80 K. Following the notation from [14], two temperature $T_{1 / 2 \mathrm{H}}$ and $T_{1 / 2 \mathrm{C}}$ corresponding to about $50 \mathrm{~mol} \%$ of the $\alpha$ and $\gamma$ phases upon heating and cooling, respectively, are equal to $T_{1 / 2 \mathrm{H}} \approx 255 \mathrm{~K}$ and $T_{1 / 2 \mathrm{C}} \approx 143 \mathrm{~K}$. The width of the hysteresis is defined as $\Delta T_{1 / 2}=T_{1 / 2 \mathrm{C}}-T_{1 / 2 \mathrm{H}}=112 \mathrm{~K}$. Note that our value of $\Delta T_{1 / 2}$ is slightly larger than those estimated from optical reflectivity $\left(\Delta T_{1 / 2}=72 \mathrm{~K}\right)$, differential scanning calorimetry $\left(\Delta T_{1 / 2}=96 \mathrm{~K}\right)$ and magnetic susceptibility $\left(\Delta T_{1 / 2}=80 \mathrm{~K}\right)$ measurements in [14].

FMS Mo K-edge XANES calculations performed for $\alpha-\mathrm{CuMoO}_{4}$ and $\gamma-\mathrm{CuMoO}_{4}$ phases are shown in Fig. 4. They are in a reasonable agreement with the experimental data. Since there are three non-equivalent molybdenum atoms in the unit cells of both molybdates [24], we have calculated also their contributions into the total XANES spectrum (see two lower panels in Fig. 4).

As one can see, the three molybdenum atoms, located in distorted tetrahedral coordination with the Mo-O distances ranged between $1.70 \AA$ and $1.86 \AA$ [24], produce slightly different XANES for $\alpha-\mathrm{CuMoO}_{4}$. At the same time, the three contributions to XANES from molybdenum atoms, located in strongly distorted octahedral environment with the Mo-O bond lengths ranged between $1.68 \AA$ and 2.49 $[24]$, are close for $\gamma-\mathrm{CuMoO}_{4}$, except for the region of the pre-edge peak. These differences are caused by an influence of outer coordination shells located above $2.5 \AA$, whose detailed analysis using reverse Monte 
Low Temperature Physics/Fizika Nizkikh Temperatur, 2018, v. 44, No.5, pp. 568-572.

Carlo approach is in progress.

\section{Conclusions}

We report the first in situ x-ray absorption spectroscopy study of the ther- mochromic phase transition between brownish-red $\gamma-\mathrm{CuMoO}_{4}$ and green $\alpha-\mathrm{CuMoO}_{4}$ in the temperature range from 10 to $300 \mathrm{~K}$. We found that the $\mathrm{Cu} \mathrm{K}$-edge XANES is weakly affected upon the phase transition because the local environment of copper atoms does not change significantly. At the same time, the analysis of the Mo K-edge XANES allowed us to follow the transition, tracing a variation of the pre-edge shoulder.

The experimental Mo K-edge XANES data were interpreted based on ab initio fullmultiple-scattering calculations. Good agreement was found between the experimental and simulated XANES spectra, and the contribution from non-equivalent molybdenum atoms in the crystallographic unit cell was estimated. It was shown that the amplitude of the pre-edge shoulder correlates with the type and the degree of distortion of molybdenum-oxygen coordination polyhedra.

A significant variation of the experimental Mo K-edge XANES upon the thermochromic transition occurs due to the change of the local environment of molybdenum atoms from distorted tetrahedral in green $\alpha-\mathrm{CuMoO}_{4}$ to distorted octahedral in brownish-red $\gamma-\mathrm{CuMoO}_{4}$. Moreover, the thermochromic transition has well evidenced hysteretic behavior, in agreement with the results of previous studies [14]. The transition from the $\gamma$-to- $\alpha$ phase occurs in the temperature range of 230-280 K upon heating, whereas the $\alpha$-to- $\gamma$ transition occurs between 120 and $200 \mathrm{~K}$ upon cooling. The width of the hysteresis was estimated to be about $112 \mathrm{~K}$.

\section{Acknowledgments}

Financial support provided by Scientific Research Project for Students and Young Researchers Nr. SJZ/2017/5 realized at the Institute of Solid State Physics, University of Latvia is greatly acknowledged. The experiment at HASYLAB/DESY was performed within the project I-20160149 EC. 
Low Temperature Physics/Fizika Nizkikh Temperatur, 2018, v. 44, No.5, pp. 568-572.

\section{References}

1. V. A. Isupov, Ferroelectrics 322, 83 (2005). doi:10.1080/00150190500315574.

2. S. Dey, R. A. Ricciardo, H. L. Cuthbert, P. M. Woodward, Inorg. Chem. 53, 4394 (2014). doi:10.1021/ic4031798.

3. D. Millers, L. Grigorjeva, S. Chernov, A. Popov, P. Lecoq, E. Auffray, Phys. Status Solidi B 203, 585 (1997). doi:10.1002/1521-3951(199710)203:2<585::AID-PSSB585>3.0.CO;28 .

4. A. Kalinko, A. Kotlov, A. Kuzmin, V. Pankratov, A. I. Popov, L. Shirmane, Centr. Eur. J. Phys. 9, 432 (2011). doi:10.2478/s11534-010-0108-7.

5. E. Auffray, M. Korjik, M. Lucchini, S. Nargelas, O. Sidletskiy, G. Tamulaitis, Y. Tratsiak, A. Vaitkeviius, Opt. Mater. 58, 461 (2016). doi:10. 1016/j.optmat.2016.06.040.

6. A. Kuzmin, V. Pankratov, A. Kalinko, A. Kotlov, L. Shirmane, A. I. Popov, Low Temp. Phys. 42,543 (2016). doi:10.1063/1.4959010.

7. A. Kalinko, A. Kuzmin, P. Roy, R. A. Evarestov, Low Temp. Phys. 42, 552 (2016). doi:10.1063/1.4959012.

8. Y. Hizhnyi, V. Chornii, S. Nedilko, M. Slobodyanik, K. Terebilenko, V. Boyko, O. Gomenyuk, V. Sheludko, Radiat. Meas. 90, 314 (2016). doi:10.1016/j.radmeas.2016.01.014.

9. A. S. Vanetsev, Y. V. Orlovskii, V. Nagirnyi, I. Sildos, L. Puust, K. Kaldvee, M. Yin, X. T. Wei, V. N. Makhov, Radiat. Meas. 90, 329 (2016). doi:10.1016/j.radmeas.2016.01.009.

10. S. Dutta, S. Som, A. K. Kunti, S. K. Sharma, V. Kumar, H. C. Swart, H. G. Visser, Nucl. Instrum. Methods Phys. Res. B 384, 76 (2016). doi:10. 1016/j.nimb.2016.08.007. 
Low Temperature Physics/Fizika Nizkikh Temperatur, 2018, v. 44, No.5, pp. 568-572.

11. Y. Zhai, M. Wang, Q. Zhao, J. Yu, X. Li, J. Lumin. 172, 161 (2016). doi:10.1016/j.jlumin.2015.11.037.

12. D. A. Spassky, N. S. Kozlova, V. Nagirnyi, A. E. Savon, Y. A. Hizhnyi, S. G. Nedilko, J. Lumin. 186, 229 (2017). doi:10.1016/j.jlumin.2017.02.048.

13. F. Rodríguez, D. Hernández, J. Garcia-Jaca, H. Ehrenberg, H. Weitzel, Phys. Rev. B 61, 16497 (2000). doi:10.1103/PhysRevB.61.16497.

14. M. Gaudon, C. Carbonera, A. E. Thiry, A. Demourgues, P. Deniard, C. Payen, J. F. Létard, S. Jobic, Inorg. Chem. 46, 10200 (2007). doi:10.1021/ic701263c.

15. M. Gaudon, P. Deniard, A. Demourgues, A. E. Thiry, C. Carbonera, A. Le Nestour, A. Largeteau, J. F. Létard, S. Jobic, Adv. Mater. 19, 3517 (2007). doi:10.1002/adma.200700905.

16. A.-E. Thiry, M. Gaudon, C. Payen, N. Daro, J.-F. Létard, S. Gorsse, P. Deniard, X. Rocquefelte, A. Demourgues, M.-H. Whangbo, S. Jobic, Chem. Mater. 20, 2075 (2008). doi:10.1021/cm703600g.

17. M. Gaudon, C. Riml, A. Turpain, C. Labrugere, M. H. Delville, Chem. Mater. 22, 5905 (2010). doi:10.1021/cm101824d.

18. I. Yanase, T. Mizuno, H. Kobayashi, Ceram. Int. 39, 2059 (2013). doi:10.1016/j.ceramint.2012.08.059.

19. W. Wen, L. Jing, M. G. White, N. Marinkovic, J. C. Hanson, J. A. Rodriguez, Catal. Lett. 113, 1 (2007). doi:10.1007/s10562-006-9003-7.

20. T. Ghorai, D. Dhak, S. Dalai, P. Pramanik, Mater. Res. Bull. 43, 1770 (2008). 
Low Temperature Physics/Fizika Nizkikh Temperatur, 2018, v. 44, No.5, pp. 568-572.

doi:10.1016/j.materresbull.2007.07.009.

21. K. S. Makarevich, N. V. Lebukhova, P. G. Chigrin, N. F. Karpovich, Inorg. Mater. 46, 1359 (2010). doi:10.1134/S0020168510120162.

22. M. Najafi, A. Abbasi, M. Masteri-Farahani, V. H. N. Rodrigues, Inorg. Chim. Acta 433, 21 (2015). doi:10.1016/j.ica.2015.04.030.

23. P. G. Chigrin, N. V. Lebukhova, A. Y. Ustinov, Kinet. Catal. 54 (2013) 76-80. doi:10.1134/S0023158413010035.

24. M. Wiesmann, H. Ehrenberg, G. Miehe, T. Peun, H. Weitzel, H. Fuess, J. Solid State Chem. 132, 88 (1997). doi:10.1006/jssc.1997.7413.

25. H. Ehrenberg, H. Weitzel, H. Paulus, M. Wiesmann, G. Wltschek, M. Geselle, H. Fuess, J. Phys. Chem. Solids 58, 153 (1997). doi:10. 1016/S0022-3697(96)00108-4.

26. G. Steiner, R. Salzer, W. Reichelt, Fresenius J. Anal. Chem. 370, 731 (2001). doi:10.1007/s002160000630.

27. T. Ito, H. Takagi, T. Asano, Chem. Mater. 21, 3376 (2009). doi:10.1021/cm901029v.

28. J. Baek, A. S. Sefat, D. Mandrus, P. S. Halasyamani, Chem. Mater. 20, 3785 (2008). doi:10.1021/cm801162j.

29. S. C. Abrahams, J. L. Bernstein, P. B. Jamieson, J. Chem. Phys. 48, 2619 (1968). doi:10.1063/1.1669492.

30. B. Ravel, M. Newville, J. Synchrotron Rad. 12, 537 (2005). doi:10.1107/S0909049505012719. 
Low Temperature Physics/Fizika Nizkikh Temperatur, 2018, v. 44, No.5, pp. 568-572.

31. Y. Joly, Phys. Rev. B 63, 125120 (2001). doi:10.1103/PhysRevB.63.125120.

32. O. Bunau, Y. Joly, J. Phys.: Condens. Matter 21, 345501 (2009). doi:10.1088/0953$8984 / 21 / 34 / 345501$.

33. L. Hedin, B. I. Lundqvist, J. Phys. C: Solid State Phys. 4, 2064 (1971). doi:10.1088/0022$3719 / 4 / 14 / 022$.

34. U. von Barth, L. Hedin, J. Phys. C: Solid State Phys. 5, 1629 (1972). doi:10.1088/0022$3719 / 5 / 13 / 012$.

35. O. Keski-Rahkonen, M. O. Krause, At. Data Nucl. Data Tables 14, 139 (1974). doi:10.1016/S0092-640X(74)80020-3.

36. A. Kuzmin, J. Purans, Proc.SPIE 2968, 180 (1997). doi:10.1117/12.266831.

37. A. Kuzmin, J. Purans, P. Parent, H. Dexpert, J. Phys. IV (France) 7, C2-891 (1997). doi:10.1051/jp4:1997267.

38. F. Rocca, A. Kuzmin, P. Mustarelli, C. Tomasi, A. Magistris, Solid State Ionics 121, 189 (1999). doi:10.1016/S0167-2738(98)00546-3.

39. A. Tougerti, E. Berrier, A.-S. Mamede, C. La Fontaine, V. Briois, Y. Joly, E. Payen, J.F. Paul, S. Cristol, Angew. Chem. 52, 6440 (2013). doi:10.1002/anie.201300538. 
Low Temperature Physics/Fizika Nizkikh Temperatur, 2018, v. 44, No.5, pp. 568-572.

Figures

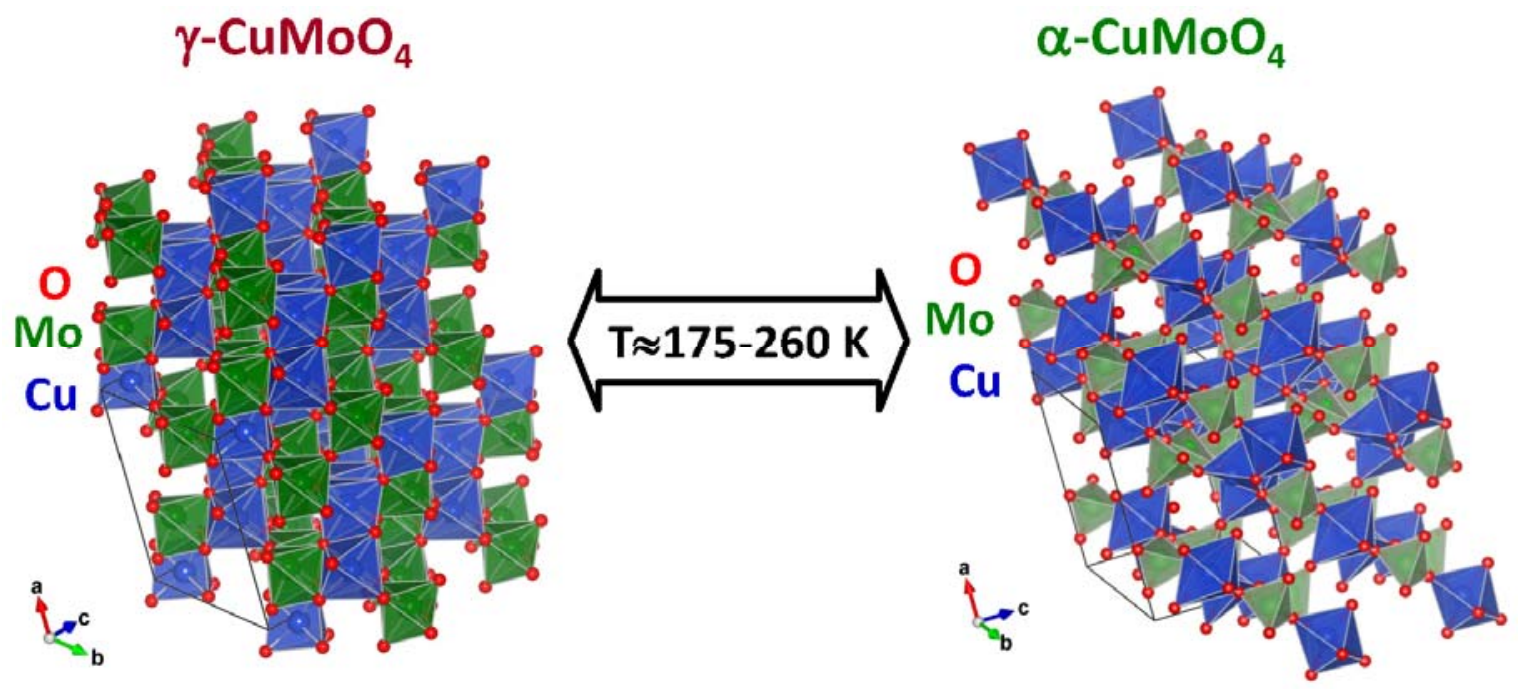

FIG. 1. Crystallographic structures of high-temperature $\alpha-\mathrm{CuMoO}_{4}$ and low-temperature $\gamma$ $\mathrm{CuMoO}_{4}$ phases [24]. 
Low Temperature Physics/Fizika Nizkikh Temperatur, 2018, v. 44, No.5, pp. 568-572.
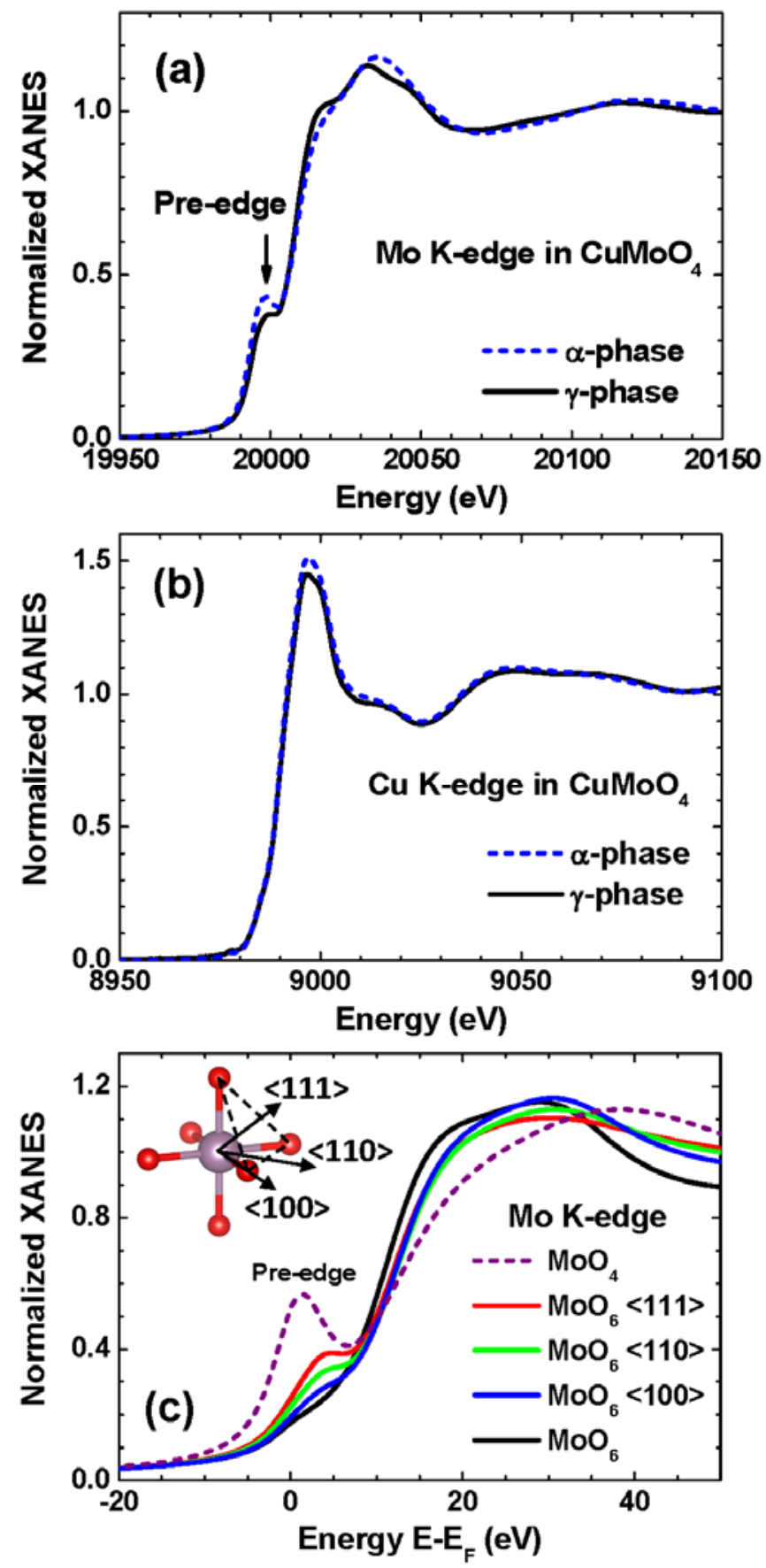

FIG. 2. (a,b) Experimental $\mathrm{Mo}$ and $\mathrm{Cu} \mathrm{K}$-edge XANES of $\alpha-\mathrm{CuMoO}_{4}($ at $300 \mathrm{~K}$ ) and $\gamma$ $\mathrm{CuMoO}_{4}\left(\right.$ at $10 \mathrm{~K}$ ) phases. (c) Calculated Mo K-edge XANES for regular $\mathrm{MoO}_{4}$ tetrahedron, distorted and regular $\mathrm{MoO}_{6}$ octahedra. Numbers in brackets indicate the direction of molybdenum ion off-center displacement. See text for more details. 
Low Temperature Physics/Fizika Nizkikh Temperatur, 2018, v. 44, No.5, pp. 568-572.

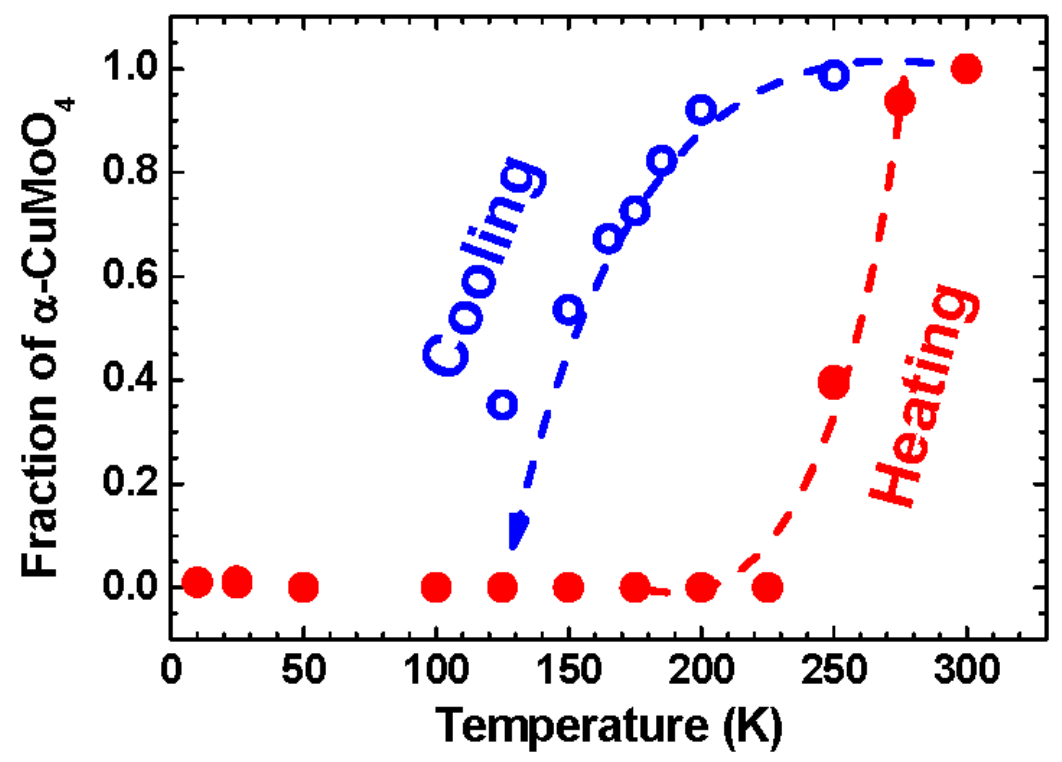

FIG. 3. Temperature dependence of the fraction of $\alpha-\mathrm{CuMoO}_{4}$ phase upon heating (solid circles) and cooling (open circles). 
Low Temperature Physics/Fizika Nizkikh Temperatur, 2018, v. 44, No.5, pp. 568-572.
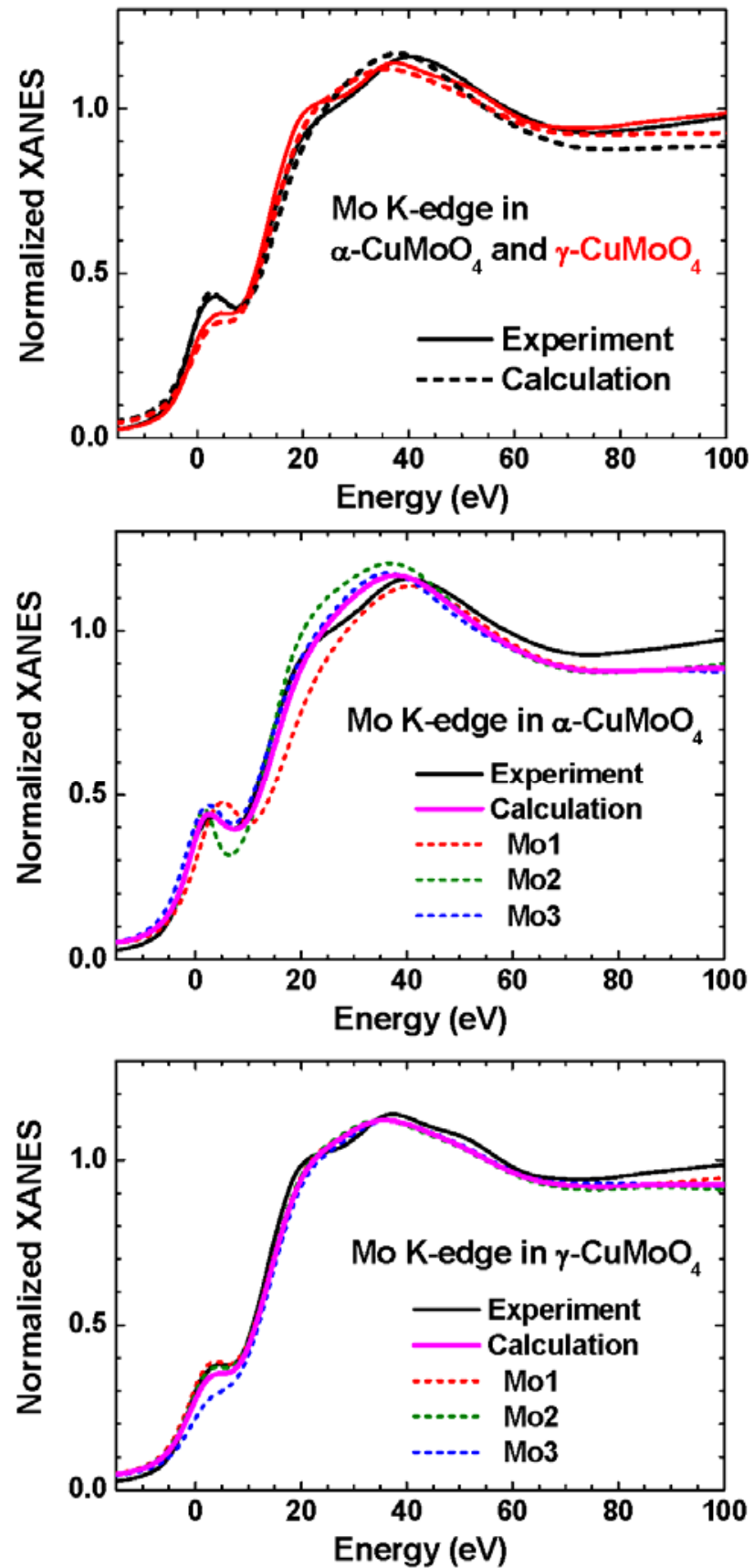

FIG. 4. Comparison of the experimental and calculated Mo K-edge XANES of $\alpha-\mathrm{CuMoO}_{4}$ and $\gamma-\mathrm{CuMoO}_{4}$ phases (upper panel). Contributions from three non-equivalent molybdenum atoms (Mo1, Mo2, Mo3) to the Mo K-edge XANES are also shown in the middle and lower panels for $\alpha-\mathrm{CuMoO}_{4}$ and $\gamma-\mathrm{CuMoO}_{4}$ phases, respectively. 